\title{
L'Idiot de la famille : repères bibliographiques
}

\section{Gilles Philippe}

\section{OpenEdition}

Journals

Édition électronique

URL : http://journals.openedition.org/recherchestravaux/246

DOI : 10.4000/recherchestravaux.246

ISSN : 1969-6434

\section{Éditeur}

UGA Éditions/Université Grenoble Alpes

\section{Édition imprimée}

Date de publication : 15 octobre 2007

Pagination : 181-186

ISBN : 978-2-84310-111-3

ISSN : 0151-1874

\section{Référence électronique}

Gilles Philippe, «L'Idiot de la famille : repères bibliographiques », Recherches \& Travaux [En ligne], 71 | 2007, mis en ligne le 15 avril 2009, consulté le 08 septembre 2020. URL : http://

journals.openedition.org/recherchestravaux/246; DOI : https://doi.org/10.4000/recherchestravaux. 246 


\section{L'Idiot de la famille : repères bibliographiques}

Ambroise C., "L'Idiot de la famille: una critica letteraria antistrutturalista », Aut-Aut, no I 37-1 39, I973, p. 85-102.

Améry C., « Die Wörter Gustave Flauberts: über Jean-Paul Sartres L'Idiot de la famille », Merkur, vol. 25, n I 2, I 97 I, p. I I 97-I 2 I 0.

Aronson R., "L'Idiot de la famille: the Ultimate Sartre? », Critical Essays on J.-P. Sartre, R. Wilcocks éd., Boston, G.K. Hall, I988, p. I I 9- I 36.

Barnes H. A., " Flaubert and Sartre on Madness in King Lear », Philosophy and Literature, vol. I , $\mathrm{n}^{\circ}$ 2, I 986, p. 2 I I-22 I.

Barnes H. A., Sartre and Flaubert, Chicago, University of Chicago Press, I 98 I.

Beauvoir S. de, Tout compte fait, Gallimard, I 972.

Bem J., «La production du sens chez Flaubert : la contribution de Sartre », La Production du sens chez Flaubert, Actes du colloque de Cerisy, UGE, « I / I 8 », I 975 , p. I 55-I 74.

Biemel W., «Sartres Vermächtnis: Die Flaubert-Deutung», Sartre, Reinbek bei Hamburg, Rowohlt, I991, p. I 59-173.

Boisdeffre P. de, "Flaubert réinventé », L’Île aux livres, Seghers, I980, p. 57-7I.

Bollème G., « Flaubert, Sartre, le roman vrai, l'histoire vécue », Études sartriennes, $\mathrm{n}^{\circ} 2-3$, I986, p. 26I-266.

Bourdieu P., Les Règles de l'art, Seuil, I 992 ; nouvelle édition, " Points », I 998 .

Briosi S., " "La storia truccata”: qualche analisi di L'Idiota della famiglia », Letteratura e società, Palermo, Palumbo, I980, p. 765-783.

—, «À la recherche de la totalité perdue », Études sartriennes, $\mathrm{n}^{\circ} 2-3$, I986, p. 24I-250. 
—, "L'immaginazione controllata: L'Idiot de la famille », Sartre critico, Bologne, Zanichelli, i 98 I, p. I I I- I 20.

Bruneau J., "Jean-Paul Sartre biographe de Flaubert », Lectures de Sartre, C. Burgelin éd., Presses Universitaires de Lyon, I986, p. I6 I-I 83.

Brunkhorst H., "Wie Man sich zu dem Macht, der Mann ist ", Sartres Flaubert Lesen: Essays zи Der Idiot der Familie, König T. éd., Reinbek bei Hamburg, Rowohlt, I980, p. 27-43.

Burgelin C., «De Sartre à Flaubert ou la genèse d'un roman vrai », RHLF, vol. $86, \mathrm{n}^{\circ} 4-5, \mathrm{I} 98 \mathrm{I}, \mathrm{p} .688-70 \mathrm{I}$.

—, «Lire L'Idiot de la famille? ", Littérature, nº 6, I 972, p. I I I- 20.

Butler J., "Desire and Recognition in Sartre's Saint-Genet and The Family Idiot vol. I », International Pbilosophical Quarterly, vol. 26, n ${ }^{\circ}$, I 986, p. 359-374.

Casanas M., «Sartre y su idiota », Concordia, n 1 7, 1990, p. 53-59.

Caute D., «The Refusal To Be God: Sartre on Flaubert», Collisions. Essays and Reviews, Londres, Quartet Books, 1974, p. 173-187.

Caws P., "Choosing Emotions: the Late Sartre and the Early Flaubert », Bulletin de la Société Américaine de philosophie de langue française, vol. 4, n 2-3, I992, p. 209-2I7.

—, "Flaubert's Laughter ", Philosophy and Literature, vol. 8, $\mathrm{n}^{\circ}$ 2, I984, p. $167-179$.

—, «L'idiot savant », Études sartriennes, n 2-3, I 986, p. 2 I-260.

—, "Sartre's Last Philosophical Manifesto », H. J. Silverman éd., Philosophers ad Non-Philosophers since Merleau-Ponty, Londres, Routledge, I988, p. IO6-I I 9 .

Clément B., «En haine de soi », Le Lecteur et son modèle, PUF, I 999, p. 17 5-255. —, «Acheminement vers la méthode : sur L'Idiot de la famille», Fondements, évolutions et persistance des théories du roman, A. Pfersmann éd., Minard, 1998, p. 239-26I.

Cohen G., "De Roquentin à Flaubert », Revue de métaphysique et de morale, vol. 8I, no I, I 976, p. I I 2-I4I.

Cohen-Solal A., "Entre Flaubert et les Maos », Sartre (1905-1980) [1985], Gallimard, «folio essais », I 999, p. 753-808.

Collins D., Sartre as Biographer, Cambridge Mass., Harvard University Press, I 980 .

Cosman C., "Translating The Family Idiot», Sartre Studies International, vol. I, $\mathrm{n}^{\circ} \mathrm{I}-2, \mathrm{I} 995, \mathrm{p} .37-44$.

Davies H., "L'Idiot des Temps modernes ", Études sartriennes, $\mathrm{n}^{\circ} 4$, I990, p. 209-220.

Digeon C., « Flaubert et le scientisme d'après Sartre », Hommage à Claude Faisant, Les Belles Lettres, I991, p. 203-22 I. 
Dörner K., «Die Wiedergeburt der Psychiatrie aus der Philosophie in Sartres Flaubert», Sartres Flaubert Lesen: Essays zu Der Idiot der Familie, König T. éd., Reinbek bei Hamburg, Rowohlt, I980, p. 60-83.

Fabre-Luce A., "Sartre par Flaubert », Revue des Deux Mondes, octobre I 972 , p. 44-6I.

Falconer G., "Sartre and/on Flaubert », Queen's Quarterly, vol. 9I, n 2, I984, p. 4I 5-424.

Farina G. et Kirchmayr R. éd., Il soggetto e l'immaginario. Percorsi nel Flaubert di Sartre, Roma, Edizioni Associate, $200 \mathrm{I}$.

—, «Dalla crisi del soggetto al progetto di sé. Un'ermeneutica del soggetto ", Il soggetto e l'immaginario. Percorsi nel Flaubert di Sartre, G. Farina Gabriella et R. Kirchmayr éd., Rome, Edizioni Associate, 2001, p. 9-39.

Frank M., «Das Individuum in der Rolle der Idioten. Die hermeneutische Konzeption des Flaubert ", Sartres Flaubert Lesen: Essays zu Der Idiot der Familie, König T. éd., Reinbek bei Hamburg, Rowohlt, I980, p. 84-ıо8.

—, "Archäologie des Individuum. Zur Hermeneutik von Sartres Flaubert», Das Sagbare und das Unsagbare, Francfort/Main, Suhrkamp, I 980, p. 36 - I I 3.

—, «Idiot ou individu ? l'intuition embryonnaire du Flaubert», Études sartriennes, $\mathrm{n}^{\circ} 2-3$, I986, p. $23 \mathrm{I}-340$.

Goldschlager A., "L'Idiot de la famille, une théorie du langage ? ", Études sartriennes, $\mathrm{n}^{\circ}$ 2-3, I986, p. I 87-198.

Gore K., "Sartre and Flaubert: from Antipathy to Empathy », Journal of the British Society for Phenomenology, vol. 4, n 2, I 973, p. I 04 - I I 2.

Grégoire J.-F., « La haine et ses corrélations dans L'Idiot de la famille», Foi et temps, vol. I $4, \mathrm{n}^{\circ}$ 5, p. $425-453$.

Grene M., " Postscript: On First Reading The Idiot of the Family », Sartre, New York, New Viewpoints, I 973, p. 265-267.

Grimm R., " Der Idiot de la famille als Herausforderungen der Literaturgeschichtsschreibung », Sartres Flaubert Lesen: Essays z $\leadsto$ Der Idiot der Familie, König T. éd., Reinbek bei Hamburg, Rowohlt, I980, p. I69-179.

Gross D., «Sartre's (Mis)reading of Flaubert's Politics», Yale French Studies, $\mathrm{n}^{\circ} 68$, I 985 , p. I 27 - I 5 I.

Guerrieri S., "Opus niger. Il nulla della parola e la parola del nulla in due momenti dell'opera sartriana: La Nausée e L'Idiot de la famille ", Il soggetto e l'immaginario. Percorsi nel Flaubert di Sartre, G. Farina Gabriella et R. Kirchmayr éd., Rome, Edizioni Associate, 200I, p. 40-I Io.

Guyaux J., «Sartre et Flaubert », La Revue générale, janvier I989, p. I9-28.

Haarscher G., "Langage et fins de l'espère : à propos de L'Idiot de la famille I», Annales de l'Institut de philosophie, Université libre de Bruxelles, I974, p. I95-2I 8. 
Halpern J., "L'Idiot de la famille », Critical Fictions. The Literary Critics of JeanPaul Sartre, New Haven, Yale University Press, I 976, p. I I 5-165.

Hart H., «Immaginazione e prassi. A proposito dell'analisi sartriana di Flaubert ", L'Immagine riflessa, $\mathrm{n}^{\circ}$ 3, I 980 , p. I 20- I 3 I.

Howells C., "Flaubert' Blind Spot: the Fetishization of Subjectivity », Situating Sartre, J.-F. Fourny et Ch. Minahen, éd., New York, St Martin Press, I 997, p. 29-38.

—, «Sartre et la déconstruction du sujet », Études sartriennes, n 5, I993, p. $217-229$.

Jopling D., "Anti-psychiatry in Sartre's The Family Idiot », Review of Existential Psychology and Psychiatry, vol. 19, n ${ }^{\circ} 2-3$, 1985 , p. I67-1 87.

Kirchmayr R., " "Legge" e "immaginario" ne L'Idiot de la famille », Il soggetto e l'immaginario. Percorsi nel Flaubert di Sartre, G. Farina Gabriella et R. Kirchmayr éd., Rome, Edizioni Associate, 200 I, p. I I I- 86.

—, "Leggere il Flaubert di Sartre », Il soggetto e l'immaginario. Percorsi nel Flaubert di Sartre, G. Farina Gabriella et R. Kirchmayr éd., Rome, Edizioni Associate, 200I, p. 5-8.

Knight D., "Sartre for Flaubertians », Neophilologus, vol. 69, n 1 , I985, p. $46-58$.

Koch G., "Zwitter-Schwestern. Weiblichkeitswahn und Frauenhass. Jean-Paul Sartres Thesen von der androgynen Kunst », Sartres Flaubert Lesen: Essays zu Der Idiot der Familie, t. König éd., Reinbek bei Hamburg, Rowohlt, I 980, p. 44-59.

König T. éd., Sartres Flaubert Lesen: Essays zu Der Idiot der Familie, Reinbek bei Hamburg, Rowohlt, I980.

—, «Von der Neurose zur absoluten Kunst », Sartres Flaubert Lesen: Essays z $u$ Der Idiot der Familie, t. König éd., Reinbek bei Hamburg, Rowohlt, I 980 , p. 44-59.

Krozova A., «Was kann man von einem Menschen wissen ?», H. Berking, Kultursoziologie - Symptom des Zeitgeistes, Würburg, Köngshausen und Neumann, 1989, p. 195-217.

La Capra D., "Flaubert, L'Idiot de la famille », A Preface to Sartre, Ithaca, Cornell University Press, I 978, p. I94-2 I 8.

Lecarme J., "Sartre et son double », NRF, n 232, I 972, p. 84-88.

Leenhardt J., «L'homme ou l'œuvre : la fonction du social dans L'Idiot de la famille», Études sartriennes, $\mathrm{n}^{\circ}$ 2-3, 1986, p. 267-278.

Le Huenen R., Perron P., «La méthode progressive-régressive. L'Idiot de la famille et la psychanalyse existentielle ", Psychanalyse et langage littéraire, J. Le Gaillot et al. éd., Nathan, I 977, p. I 57-1 86. 
Lippitz W., «Das Werden eines Ich. Biografische Rekonstruktion frühkindlicher Sozialisation am Beispiel von Sartres Idiot der Familie », Zeitschrift für Sozialisationforschung und Erziehungssoziologie, vol. 8, nº I, I988, p. $40-53$.

Maler E., Sartres Individualhermeneutik, Vienne-Munich, Fink, I 986.

Marchand J., "Sartre, Flaubert et Dieu », Raison présente, n 33, I 975, p. $65-77$.

Maurel J., "Sartre dans Flaubert», Autrement, n 20, 200 I, p. I 3 I-I 45.

McBride W., "Method and Madness in The Family Idiot», D. Ihde et H. J. Silverman éd., Albany, SUNY Press, I985, p. I42-i60.

McKenna R., "Sartre's Flaubert: a Contribution to Psychoanalytic Theory? ", Sartre. An Investigation of Major Themes, S. Glynn éd., Aldershot, Avebury, 1987, p. 86-103.

Mitzman A., «Sartres Flaubert», Psyche, Heidelberg, vol. 35, I98 I, p. 268-298.

Mouchard C., «Un roman vrai ?", Critique, n 295, I97 I, p. I029-1049.

Müller-Lisner A., Sartre als Biograph Flauberts. Zu Zeilen und Methoden von L'Idiot de la famille, Bonn, Bouvier Verlag, 1977.

Neuschafer Anne, Zur Rezeption des Idiot de la famille, Literarische Diskurse des Existentialismus, H. Harth et V. Roloff éd., Tübingen, Stauffenburg Verlag, I986, p. I03-II 4.

Noudelmann F., Sartre. L'incarnation imaginaire, L'Harmattan, I 996.

Noudelmann F., Philippe G. éd., Dictionnaire Sartre, Champion, 2004.

Oehler D., «Zur gesellschaftlichen Standort der Neurose-Kunst. Sartres L'Idiot de la famille und Flauberts Éducation sentimentale: Versuch einer vergleichenden Lektüre ", Sartres Flaubert Lesen: Essays zu Der Idiot der Familie, König T. éd., Reinbek bei Hamburg, Rowohlt, I 980, p. I49-190.

Pacaly J., Sartre au miroir, Klincksieck, I 980, p. 31 5-428.

Pinto E., «La névrose objective chez Sartre », Les Temps modernes, n 339 , I 974, P. 35-76.

Polan D., " A Vertigo of Displacement: an Introduction to the Sartrean Spectacle of L'Idiot de la famille ", Dalhousie French Review, vol. 64, n 2, I984, p. 354-375.

Pucciani O., «Sartre and Flaubert as Dialectic », The Philosophy of Jean-Paul Sartre, P. A. Schilpp éd., La Salle, Open Court, I98 I, p. 495-538.

Rizk H., «Flaubert : individu et totalisation (L'Idiot de la famille, tome III)», Alter, $\mathrm{n}^{\circ}$ Io, 2000, p. 249-264.

Roloff V., «Von der "psychanalyse existentielle” zur Sozialgeschichte der Literatur ", Lendemains, vol. 5, n 17-1 8, I980, p. 109-I 24.

Roudiez L. S., «Flaubert, for Instance ? », French Review, vol. 55, n 7, 1982 , p. 68-78.

Rubino G., "Il romanzo critico », Jean-Paul Sartre, Florence, La Nuova Italia, I 983 , p. I 37 -I 49 . 
Schulten M., Jean-Paul Sartres L'Idiot de la famille. Ein methodisches Modell der Dichterbiographie, Berne, Peter Lang, I 99I.

Scriven M., Sartre's Existential Biographies, Londres, Macmillan, I 984.

Sicard M., "Flaubert avec Sartre ", La Production du sens chez. Flaubert. Actes du colloque de Cerisy, UGE, «Io/I 8 », I975, p. I75-1 89.

—, «Flaubert», Sartre. Catalogue de l'exposition de la Bibliothèque nationale de France, M. Berne éd., Éditions de la BNF-Gallimard, 2005, p. 266-269.

—, «Jean-Paul Sartre, L'Idiot de la famille », Cabiers du chemin, $\mathrm{n}^{\circ}$ 27, 1976 , p. I 38 -I 58.

—, Essais sur Sartre. Entretiens avec Sartre, Galilée, I989 ("Le continent Flaubert», p. 59-I 8I).

Sollich K.-M., «Der Idiot de la famille: Efeu Revisited », Das Sartre-Jahrbuch, R.E. Zimmermann éd., vol. II, Münster, Verlag Westfälisches Bampfboot, I99I, P. I 30-I 37.

Sportelli S., "Il metodo progressivo-regressivo applicato: L'Idiot de la famille», Sartre e la psicanalisi, Bari, Dedalo, I 98 I, p. I 86-2 I I.

Tamassia P., " "L'imaginaire réalisé": il potere eversivo dell'immaginario (da L'Imaginaire a L'Idiot de la famille)", Il soggetto e l'immaginario. Percorsi nel Flaubert di Sartre, G. Farina Gabriella et R. Kirchmayr éd., Rome, Edizioni Associate, 2001, p. I 87-203.

Tognonato C., «Immaginare Flaubert. Una questione di metodo », Il soggetto e l'immaginario. Percorsi nel Flaubert di Sartre, G. Farina Gabriella et R. Kirchmayr éd., Rome, Edizioni Associate, 200 I, p. 204-236.

Utti K., "Sartre L'Idiot de la famille: a Contribution to the Theory of Narrative Style », Romanic Review, n 68, I 977, p. 2 1 7-234.

Verstraeten P., "Sartre et son rapport à la névrose objective », Autour de Jean-Paul Sartre, P. Verstraeten éd., Gallimard, I98 I, p. I9-54.

—, «Sens et structure de Saint Genet et de L'Idiot», Études sartriennes, $\mathrm{n}^{\circ}{ }_{2-3}$, 1986, p. 199-230.

—, "The Negative Theology of Sartre's Flaubert », Yale French Studies, $\mathrm{n}^{\circ} 68, \mathrm{I} 985$, p. I 5 2-164.

Volpi R., « Riflessioni sul Sartre critico: continuità fra L'Idiota della famiglia e la filosofia del primo Sartre », Dimensioni, n 39, I 986, p. 17-29.

Wannicke R., Sartres Flaubert: zur Misanthropie der Einbildungskeraft, Berlin, Reimer, I990. 